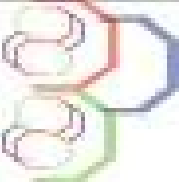

Journal of Applied Biosciences 67:5210-5218

ISSN 1997-5902

\title{
Évaluation de la qualité microbiologique du chinchard (Trachurus trachurus) au cours du processus de fumage traditionnel
}

\author{
René G. DEGNON*, Valentin AGOSSOU, Euloge S. ADJOU, Edwige DAHOUENON-AHOUSSI, Mohamed \\ M. SOUMANOU, Dominique C.K. SOHOUNHLOUE
}

Laboratoire d'Etude et de Recherche en Chimie Appliquée. Ecole Polytechnique d'Abomey-Calavi, Université d'Abomey-Calavi, Bénin, 01 BP : 2009 Cotonou, Bénin.

*Adresse pour correspondance : gnimabou2000@yahoo.fr

Original submitted in on $6^{\text {th }}$ June 2013 Published online at www.m.elewa.org on $30^{\text {th }}$ July 2013.

https://dx.doi.org/10.4314/jab.v67i0.95042

\section{RESUME}

Objectifs: La présente étude vise à identifier les sources de contamination du poisson Chinchard (Trachurus trachurus) au cours du processus de fumage traditionnel.

Méthodologie et Résultats: Cette étude a été réalisée à partir d'une enquête semi-structurée, couplée à un échantillonnage tout au long du processus de fumage et suivi d'analyses microbiologiques. Les Germes Aérobies Mésophiles, les Coliformes totaux et thermotolérants, les Anaérobies Sulfito-Réducteurs, Escherichia coli, Staphylococcus aureus, les Levures et Moisissures et Salmonella spp. sont les principaux microorganismes qui sont recherchés dans les échantillons de poissons analysés. Les résultats de l'enquête ont révélé des insuffisances au niveau des règles d'hygiène lors de la manipulation des poissons par les transformatrices. Les charges microbiennes obtenues par étape étaient significativement différentes, avec une contamination plus élevée après le fumage et la mise en vente. La qualité microbiologique de la plupart des poissons analysés n'était pas satisfaisante. La présence des levures et moisissures $(76,7 \%)$ d'Anaérobies Sulfito-Réducteurs (56,7\%), de coliformes fécaux (33,3\%) et d'Escherichia coli $(26,7 \%)$ a été notées dans les échantillons. Par contre, les échantillons analysés n'ont pas été contaminés par les bactéries potentiellement pathogènes telles que les Salmonella spp. et Staphylococcus aureus.

Conclusion et application: Une attention particulière visant essentiellement la réduction de la contamination microbienne des poissons doit être accordée à cette filière. L'application rigoureuse des règles d'hygiène tout au long du processus de fumage et de la mise en vente réduiraient de façon significative la flore de contamination des poissons en augmentant leur durée de conservation.

Mots clés: Chinchard, fumage, qualité microbiologique, Bénin.

Evaluation of the microbiological quality of mackerel (Trachurus trachurus) during the traditional smoking process

ABSTRACT 
Objectives: This study aims to identify the sources of contamination of fish mackerel throughout the traditional smoking process.

Methodology and Results: This study was conducted using a semi-structured survey, coupled with sampling throughout the smoking process monitoring and microbiological analysis. Total flora, coliforms, Anaerobic Sulfito-reducers, Escherichia coli, Staphylococcus aureus, Yeast and Mould and Salmonella spp. are the main microorganisms that are isolated in the analyzed fish. The results revealed major deficiencies in hygiene during the process. Microbial contamination obtained during processing step was significantly different, with higher contamination after smoking and sale. The microbiological quality of most fish analyzed was not satisfactory, with the presence of yeasts and molds (76.7\%), Anaerobic Sulfito-reducers (56.7\%), fecal coliforms (33.3\%) and Escherichia coli (26.7\%). However, potentially pathogenic bacteria such as Salmonella spp. and Staphylococcus aureus were not detected in any of the samples analyzed.

Conclusion and Application: More attention aim at reducing the microbial contamination of smoked fish should be paid to this sector. The strict application of hygiene throughout the smoking process and the sale would significantly reduce the contamination of fish by improving their storage.

Keywords: mackerel, smoked, microbiological quality, Benin.

\section{INTRODUCTION}

Le poisson et ses produits dérivés jouent un rôle considérable dans l'alimentation des populations de l'Afrique de l'Ouest FAO (2000). Le poisson est une denrée alimentaire de haute valeur nutritive mais très périssable. II constitue aussi un complément précieux dans les régimes alimentaires pauvres en protéines, vitamines et sels minéraux essentiels. Au Bénin, la pêche tient une place relativement importante dans l'équilibre socio-économique national car elle fait vivre environ 500.000 personnes et contribue pour $3 \%$ au PIB (Tossou, 2010). Cependant, la conservation du poisson, notamment dans les pays chauds, est difficile en raison du manque d'infrastructures de conservation adéquates et du fait des conditions climatiques et d'environnement qui concourent à sa dégradation en quelques heures (Anihouvi et al., 2005). Au Bénin, et à l'instar des autres pays de la sous-région ouest, les pertes post-capture sont estimées à environ 20 $\%$ (Anihouvi et al., 2005). Ainsi, pour limiter ces pertes, le fumage constitue l'une des principales méthodes de conservation du poisson au Bénin et se fait encore de façon traditionnelle (Anoh, 1998). Depuis quelques années, la salubrité des poissons constitue un problème de santé publique, car les germes pathogènes et ou les contaminants chimiques présents dans les poissons fumés font peser de sérieuses menaces sur la santé des consommateurs (OMS, 2003). D'autres préoccupations peuvent advenir de la contamination de cette denrée par les moisissures, les anaérobies sulfito-réducteurs et les Staphylocoques qui, dans certaines conditions, peuvent secréter des toxines à pouvoir hépatotoxique (Gallot et Frémy, 2006 ; AFSSA, 2009). Le fumage est l'une des premières techniques de transformation traditionnelle du poisson au Bénin. II concerne aussi bien le poisson congelé importé que le poisson frais de la pêche locale. Les poissons fumés sont destinés à la consommation locale avec exportation d'une petite quantité vers les pays limitrophes tels le Togo, le Nigéria, le Burkina Faso et le Niger (Gbaguidi et Fiogbé, 1999). Au cours du processus de fumage, la fumée constitue un facteur essentiel. Ces constituants exercent des actions sur le poisson fumé, notamment sur ses qualités organoleptiques et sur la flore d'altération du poisson. L'action bactérienne est cependant sélective. Mais l'effet de la fumée est plus marquant sur Clostridium botulinum. Le chinchard fumé est un produit très consommé au Bénin à cause de sa disponibilité et de son accessibilité. Cette denrée alimentaire est produite de façon artisanale par les femmes des ethnies spécialisées dans la pratique des activités de pêche au Bénin, notamment les Toffins. Cependant, malgré les nombreux efforts fournis dans la conservation du poisson à travers le fumage, le problème subsiste toujours à cause du caractère 
périssable des poissons fumés suite à une prolifération microbienne poussée. Ainsi, la présente étude vise à évaluer la qualité microbiologique des poissons chinchard fumés tout au long de la chaine

\section{MATERIEL ET METHODES}

Enquête : Les enquêtes se sont déroulées sous forme d'entretien semi structurés et d'observation des acteurs au travail. Deux sites de fumage ont été investigués. Le choix des sites s'est fait en tenant compte d'une part à la présence importante de transformatrices et d'autre part du fait que ces sites représentent les grandes zones de fumage qui alimentent le marché Dantokpa (Sud-Bénin). De plus, le chinchard fait partie des espèces de poisson les plus fumés sur ces sites. La collecte des données s'est faite sur la base de questionnaire préétabli. Au total, soixante sept (67) acteurs composés de transformatrices et de revendeuses ont été interviewés dans les zones d'enquête ciblées. Les critères prioritaires de sélection étaient : être propriétaire d'une unité de transformation et être consentante. Cette enquête vise essentiellement à évaluer les différentes étapes de la chaine de production de poissons chinchard fumés au Bénin et à identifier les pratiques qui nuisent à la qualité des poissons fumés et qui risquent aussi de constituer un danger pour le consommateur.

Echantillonnage : Dans le but d'effectuer un suivi de qualité des poissons depuis la réception des poissons frais jusqu'à l'obtention des poissons fumés et leur mise en vente, quatre zones spécifiques ont été choisies pour l'échantillonnage. II s'agit de l'échantillonnage des poissons frais à la réception, après les opérations de prétraitements, après l'opération de fumage et enfin après trois (03) heures d'exposition des poissons fumés dans les conditions de vente. Chaque échantillon est constitué de vingt (20) unités de poisson. Le prélèvement des échantillons de poissons pour les analyses sensorielles et microbiologiques a été effectué dans des de transformation jusqu'à la mise en vente, afin d'identifier les sources potentielles de contamination ainsi que la flore microbienne associée.

conditions aseptiques : des gants stériles en latex sont utilisés pour la protection des mains lors des prélèvements; les échantillons de poissons sont collectés et emballés dans des sachets stériles et conditionnés dans une glacière portative de type "ESKIMO". L'ensemble du matériel de prélèvement est préalablement stérilisé avec du coton imbibé d'alcool à $90^{\circ} \mathrm{C}$.

Analyses microbiologiques : Les échantillons prélevés ont été évalués en recherchant par des méthodes standards les paramètres microbiologiques de qualité. II s'agit de la flore mésophile totale à $30^{\circ} \mathrm{C}$ (germes totaux ; NF V08-051), des coliformes totaux, des coliformes thermotolérants et Escherichia coli (NF ISO 4831) des Staphylocoques (Staphylococcus aureus) à $37^{\circ} \mathrm{C}$ (NF EN ISO 6888-1), des Salmonelles (NF V 08 - 052), des levures et moisissures (ISO 7954) et des spores d'Anaérobies Sulfito-réducteurs (NF V 08-061). Cette évaluation a été réalisée en utilisant comme supports, les techniques standards d'analyses rapportées par Joffin et Joffin (2003). Les milieux de cultures et réactifs utilisés proviennent des Laboratoires BioMérieux et Diagnostics Pasteur. L'interprétation des résultats a été faite suivant un plan à deux classes en référence aux critères microbiologiques pour les produits animaux frais (guide législatif et réglementaire français, $N^{\circ} 8155$ du 12 décembre 2000), fixant le seuil de tolérance à $M=10^{3}$ UFC/g ou ml pour la flore totale; à $10 \mathrm{UFC/g}$ ou $\mathrm{ml}$ pour les coliformes fécaux ; $2 \mathrm{UFC/g}$ ou $\mathrm{ml}$ pour les bacilles sulfito-réductrices et à l'absence dans $25 \mathrm{~g}$ de produit analysé pour les salmonelles. 


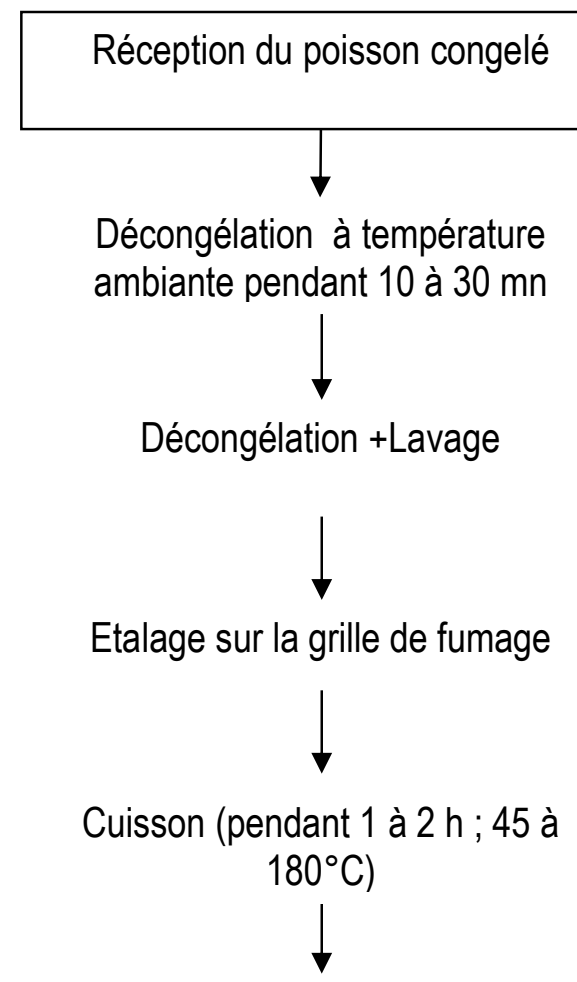

Fumage proprement dit (pendant 30

$\mathrm{mn}$ à $1 \mathrm{~h} ; 60$ à $\left.70^{\circ} \mathrm{C}\right)$

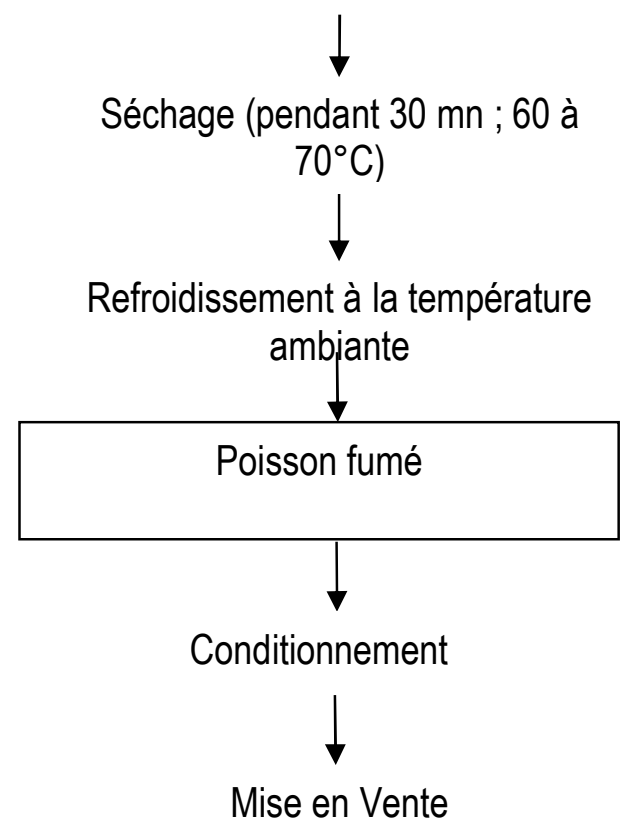

Figure 1 : Technologie traditionnelle de fumage du poisson chinchard au Sud du Bénin 
Préparation de la suspension mère : $25 \mathrm{~g}$ de chaque échantillon ont été prélevés et broyés de manière aseptique. $225 \mathrm{ml}$ d'eau peptonnée tamponnée ont été ajoutées au broyat et le mélange est homogénéisé au stomacher. A partir de cette suspension-mère des dilutions décimales ont été effectuées.

- Numération de la flore totale: Elle a été réalisée par un ensemencement dans la masse. Un (1) $\mathrm{ml}$ de la suspension mère et de ses dilutions décimales en duplicata ont été ensemencés dans la Gélose Plate Count Agar (PCA) en surfusion. L'incubation a été effectuée à $30^{\circ} \mathrm{C}$ pendant $72 \mathrm{~h}$, puis le dénombrement et la moyenne des germes en Unité Formant Colonie (UFC) $/ g$ d'échantillon analysé ont été faits selon la méthode spécifiée par la norme NF V08-051.

- Numération des levures et moisissures : Des aliquotes de $0,1 \mathrm{ml}$ de la suspension mère et de ses dilutions décimales ont été ensemencées en surface sur la gélose Sabouraud au Chloramphénicol intialement préparée et coulée dans des boîtes de pétrie de $9 \mathrm{~cm}$ de diamètre. Le dénombrement des colonies blanches ou colorées, lisses et crémeuses de levures et des moisissures sous forme poudreuse a été effectué après 5 jours d'incubation à $25^{\circ} \mathrm{C}$ selon la norme ISO 7954 .

- Recherche des coliformes totaux et fécaux: Les coliformes totaux sont recherchés selon la méthode NPP décrite par la norme NF ISO 4831. La recherche des coliformes thermotolérants est effectuée par comptage des colonies obtenues à $44{ }^{\circ} \mathrm{C}$ selon la méthode spécifiée par la norme NF V 08-060.

- Recherche des Staphylocoques : La technique d'étalement en surface de $0,1 \mathrm{ml}$ d'inoculum (échantillon et dilutions décimales) sur le milieu Baird Parker complet réalisée en duplicata a été utilisée. L'incubation des milieux ensemencés a été faite à $37{ }^{\circ} \mathrm{C} / 48 \mathrm{H}$. Les colonies caractéristiques noires brillantes entourées

\section{RESULTATS}

Les résultats de l'enquête : Les résultats des enquêtes semi-structurés réalisés au niveau des différents sites de fumage investigués sont présentés par partie, en tenant compte des différentes communautés transformatrices de poissons, les espèces de poisson couramment fumées et les conditions de fumage des poissons observées sur les sites investigués. Parmi les transformatrices enquêtées, on y trouve plusieurs ethnies. La majeure partie est constituée des Toffins suivi des Fons, des Minas, et des Wla. Cette communauté de transformatrices de poisson se situe dans une tranche d'âge de 20-65ans avec une moyenne d'âge de 32 ans. La plupart d'entre-elle (85\%) d'halo clair ont été dénombrées puis repiquées sur Chapman suivis de la coloration de Gram. Parallèlement d'autres colonies ont été triturées dans $5 \mathrm{~mL}$ de Bouillon Coeur Cervelle $(\mathrm{BCC})$ et incubées à $37^{\circ} \mathrm{C} / 24$ heures pour le test de la coagulase. La réalisation du test a consisté au mélange dans un rapport de 1/3 respectivement pour le BCC ensemencé et le sérum de lapin, le tout a été incubé à $37^{\circ} \mathrm{C} / 6$ heures; la première lecture a été faite 3 heures après. Les tubes positifs correspondent à une prise en masse du contenu. La méthode utilisée est celle décrite par la norme NF EN ISO 6888-1.

- Recherche des spores d'anaérobies sulfitoréducteurs: La recherche des spores d'Anaérobies Sulfito-réducteurs permet d'évaluer les risques de toxiinfection à Clostridium spp. Le milieu de culture utilisé est Tryptone Sulfite Néomycine (TSN). La méthode utilisée est le dénombrement en anaérobiose des bactéries sulfito-réducteurs décrite par la norme NF V 08-061.

- Recherche des Salmonella: La recherche des Salmonella dans les aliments comporte des étapes essentielles à savoir: le préenrichissement, l'enrichissement, l'isolement et la confirmation. La méthode utilisée pour cette recherche est celle spécifiée par la norme NF V 08-052.

Analyses statistiques: Les résultats ont été analysés par la méthode de variance (ANOVA) à l'aide du logiciel STATISTICA (Stat., Soft, Inc, 1995). La comparaison des moyennes est effectuée par le test de la plus petite différence significative LSD (Least Significant Difference). Cette méthode d'analyse consiste à chercher les moyennes qui diffèrent significativement les unes des autres. Les différences sont significatives lorsque $\mathrm{P}<0.05$.

est analphabète. Toutes les transformatrices utilisent la méthode de fumage traditionnel et commercialisent principalement leur produit au marché Dantokpa. Près de $66,7 \%$ des transformatrices réalisent principalement le fumage du poisson chinchard (Trachurus trachurus) décongelé. Les autres ethnies $(33,3 \%)$ réalisent majoritairement le fumage de Sarotherodon melanotheron, et du Tilapia guineensis. La plupart des transformatrices enquêtées achètent les poissons chinchards congelés à des auprès des poissonneries dont les elles ignorent parfois les sources d'approvisionnement. Le fumage des poissons se fait le 
jour même de l'achat des poissons. Le fumage traditionnel à chaud sans salaison, ni éviscération des poissons est le type de fumage utilisé. Selon $66,7 \%$ des transformatrices, c'est essentiellement l'eau de puits ou du Lac qu'elles utilisent. De même, les transformatrices rencontrés sur les sites de fumage ignorent ou ne respectent pas les règles élémentaires lors de la manipulation d'aliments destinés à la consommation publique. Sur ces sites, le fumage s'effectue souvent en plein air. Tous les types de bois et sous-produits divers sont utilisés pour la production de la fumée. Comme sous-produits utilisés, on distingue des épluchures de canne à sucre, de la sciure de bois, le papier, des épluchures de manioc, ce qui rend très variable la composition de la fumée utilisée pour le fumage sur les sites enquêtés.

Qualité microbiologique des échantillons de poissons prélevés: Les principaux microorganismes dénombrés dans les 80 échantillons de poissons prélevés sur les deux sites de fumage sont consignés dans les tableaux 1, 2, 3, 4. Les résultats obtenus au niveau du premier site de prélèvement (Tableaux 1 et 2) montrent que l'incidence de la flore d'altération et celle des germes indicateurs de contamination dans les poissons prélevés (Tableau 1) varie en fonction des différentes étapes du processus de fumage. En effet, la flore totale (Germes Aérobies Mésophiles) initiale de 9,4.106 UFC/g diminue à $6,7.10^{6} \mathrm{UFC} / \mathrm{g}$ après les opérations de prétraitement, puis à $5,4.10^{6} \mathrm{UFC} / \mathrm{g}$ après le fumage proprement dit. Ces résultats indiquent l'effet des différents traitements sur la réduction de la flore microbienne du poisson, car au cours des traitements technologiques, le dénombrement de la flore totale permet de juger de l'incidence des diverses opérations (Diouf, 1992). Ces mêmes observations sont faites au niveau des coliformes totaux $\left(1,5 \cdot 10^{1} \mathrm{UFC} / \mathrm{g}\right.$ à $\left.0,0 \mathrm{UFC} / \mathrm{g}\right)$, des levures $\left(6,3.10^{3} \mathrm{UFC} / \mathrm{g}\right.$ à $\left.1,1 \cdot 10^{3} \mathrm{UFC} / \mathrm{g}\right)$ et des moisissures $\left(2,1 \cdot 10^{3} \mathrm{UFC} / \mathrm{g}\right.$ à $\left.1.10^{1} \mathrm{UFC} / \mathrm{g}\right)$. Ces différents résultats montrent que les prétraitements ainsi que le fumage proprement dit, réduisent de façon significative $(p<5 \%)$ la flore d'altération ainsi que les germes indicateurs de contamination divers. La contamination des poissons par les Coliformes thermo-tolérants peut constituer un problème de santé publique, car ces germes peuvent entrainer la production de l'histamine, une amine biogène résistante à la chaleur et toxique pour l'homme (Huss, 2000). Les résultats du dénombrement de Staphylococcus aureus dans les échantillons sont conformes à ceux obtenus par Djinou (2001) et Goueu (2006). Cette conformité obtenue pourrait s'expliquer par le fait que l'espèce de poisson utilisée (Trachurus trachurus) est soumise au contrôle à l'embarquement et au débarquement recommandé par la règlementation. L'absence de cette flore indique le respect de l'hygiène des transformatrices. L'analyse des résultats montre également que le fumage a un effet plus prononcé sur les coliformes que sur la flore fongique. Cette forte activité antibactérienne pourrait être liée à la température de fumage, au pouvoir germicide de la fumée ou à une synergie d'action. Lors du fumage, les particules de fumée absorbées par le poisson freinent surtout le développement bactérien à la surface du produit. La chaleur du feu sèche le poisson et la viande, et si la température est assez élevée, la chair cuit. Cela prévient l'altération par les bactéries et par les enzymes. C'est le séchage et la cuisson de la chair pendant le fumage qui jouent le principal rôle de conservation. Au cours de ce traitement, on observe deux phénomènes simultanés: Une déshydratation par entraînement (le poisson étant placé dans un courant d'air chaud) et une action antiseptique aromatisante et colorante de la fumée (CTA, 1990). Ces observations sont similaires à celles de Dègnon et al., (2013), qui a également noté une décroissance de la charge microbienne lors du fumage des crevettes (Penaeus spp.). On note également une absence d'E. coli et de Staphylococcus aureus dans les échantillons de poissons frais, qui pourrait se justifier par le fait que ces germes sont respectivement témoins de contamination fécale et de contamination cutanéomuqueuse et ne pourraient provenir que des manipulateurs (Untermann, 1998). L'absence de ces germes dans les poissons après le prétraitement et l'opération de fumage indique également le respect des bonnes pratiques d'hygiène par les productrices lors des opérations de fumage du poisson. Cependant, la présence de ces germes dans les échantillons après exposition dans les conditions de vente, indique d'une recontamination certaine des échantillons due aux conditions de vente de ces poissons dans les marchés. Ces résultats sont également confirmés par l'augmentation de la flore totale ainsi que la flore fongique dans ces échantillons. Cette recontamination des échantillons résulterait du faible niveau d'hygiène au niveau de certains acteurs de la filière. De même, l'exposition des poissons pour la vente pourrait également constituer une source de contamination. Ces mêmes résultats sont également obtenus au niveau des aliments vendus dans la rue et ces germes identifiés dans ces aliments sont majoritairement les entérobactéries et les staphylocoques. Selon W.H.O 
(1998), les données épidémiologiques en milieu hospitalier montrent une prévalence de $19 \%$ des maladies diarrhéiques dont $70 \%$ des cas sont d'origine alimentaire. Les causes sont essentiellement liées aux mauvaises conditions de transformation et de vente (Leclerc et al., 2002). L'absence dans les échantillons de germes potentiellement pathogènes, notamment les salmonelles (Tableau 2), est conforme aux résultats rapportés par Djinou, (2001), Goueu (2006) et Oulaï et al. (2007). La conformité des échantillons se justifie par les contrôles sanitaires auxquels les poissons utilisés sont soumis avant leur entrée sur le territoire béninois. Ces résultats montrent que la consommation des poissons fumés ne présente pas un risque sanitaire et sont de qualité microbiologique satisfaisante par rapport aux exigences normatives (guide législatif et réglementaire français, $N^{\circ} 8155$ du 12 décembre 2000). En effet, les bactéries du genre Salmonella et principalement S.tiphy et $S$. paratiphy possèdent de nombreux facteurs de virulence: présence de pili, production de toxines, capacité à survivre dans les macrophages et présence de plasmide de virulence (Sutra et al., 1998) et sont associées principalement aux toxi-infections alimentaires collectives (TIAC). Par contre au niveau des Anaérobies Sulfito-Réducteurs, on remarque une destruction de cette flore après le fumage, confirmant ainsi l'efficacité du fumage sur les Clostridium spp. Cependant, les mauvaises conditions d'exposition de cette denrée alimentaire pourraient entrainer une recontamination et une prolifération microbienne à cause du potentiel nutritionnel du poisson.

Tableau 1 : Flore d'altération et germes indicateurs de contamination microbienne (ufc/g) dans les poissons prélevés à différentes étapes du processus de fumage (Site1)

\begin{tabular}{|l|l|c|c|c|c|c|c|}
\hline Etapes de collectes & $\begin{array}{l}\text { Flore } \\
\text { totale }\end{array}$ & $\begin{array}{l}\text { Coliformes } \\
\text { totaux }\end{array}$ & $\begin{array}{l}\text { Coliformes } \\
\text { fécaux }\end{array}$ & E.coli & $\begin{array}{l}\text { Staphylococcus } \\
\text { aureus }\end{array}$ & Levures & Moisissures \\
\hline Réception & $9,4.10^{6} \mathrm{a}$ & $1,5.10^{1} \mathrm{a}$ & - & - & - & $6,3.10^{3} \mathrm{a}$ & $2,1.10^{3}$ \\
\hline Prétraitements & $6,7.10^{6} \mathrm{~b}$ & - & - & - & - & $1,5.10^{4} \mathrm{~b}$ & $10^{1}$ \\
\hline Fumage & $5,4.10^{6} \mathrm{C}$ & - & - & - & - & $1,1.10^{3} \mathrm{c}$ & $10^{1}$ \\
\hline Mise en vente & $2,4.10^{6} \mathrm{~d}$ & $1,4.10^{3} \mathrm{a}$ & $1,5.10^{1}$ & 4,0 & - & $7,6.10^{3} \mathrm{~d}$ & $3,2.10^{2}$ \\
\hline $\begin{array}{l}\text { Critères } \\
\text { microbiologiques } \\
\text { (AFNOR 2000) }\end{array}$ & $10^{3}$ & 10 & 10 & 10 & 10 & 10 & 10 \\
\hline
\end{tabular}

Les chiffres portant la même lettre dans la même colonne ne sont pas significativement différents $(p<0,05), \quad$ - : Absence.

Tableau 2 : Flore potentiellement pathogène (ufc/g) dans les échantillons de poissons prélevés à différentes étapes du processus de fumage (Site1)

\begin{tabular}{|l|c|c|}
\hline Etapes de collectes & Anaérobies Sulfito Réducteurs & Salmonella spp. \\
\hline Réception & $2,0 \mathrm{a}$ & - \\
\hline Prétraitements & $2,0 \mathrm{a}$ & - \\
\hline Fumage & - & - \\
\hline Mise en vente & $1,0 \mathrm{a}$ & - \\
\hline Critères microbiologiques (AFNOR 2000) & $\mathbf{0 2}$ & Absence $/ \mathbf{2 5 g}$ \\
\hline
\end{tabular}

Les chiffres portant la même lettre dans la même colonne ne sont pas significativement différents $(p<0,05)$, - : Absence. 
Tableau 3 : Flore d'altération et germes indicateurs de contamination microbienne (ufc/g) dans les poissons prélevés à différentes étapes du processus de fumage (Site2)

\begin{tabular}{|l|c|c|c|c|c|c|c|}
\hline Etapes de collectes & $\begin{array}{l}\text { Flore } \\
\text { totale }\end{array}$ & $\begin{array}{c}\text { Coliformes } \\
\text { totaux }\end{array}$ & $\begin{array}{c}\text { Coliformes } \\
\text { fécaux }\end{array}$ & E.coli & $\begin{array}{c}\text { Staphylococcus } \\
\text { aureus }\end{array}$ & Levures & Moisissures \\
\hline Réception & $3,6.10^{4} \mathrm{a}$ & $2,0.10^{2} \mathrm{a}$ & - & - & - & $8.10^{1} \mathrm{a}$ & $10^{1} \mathrm{a}$ \\
\hline Prétraitements & $1,0.10^{5} \mathrm{~b}$ & $2,0.10^{2} \mathrm{a}$ & - & - & - & $5,5.10^{1} \mathrm{a}$ & - \\
\hline Fumage & $8,0.10^{2} \mathrm{c}$ & $4,0 \mathrm{~b}$ & - & - & - & - & $1,6.10^{2} \mathrm{a}$ \\
\hline Mise en vente & $1,4.10^{6} \mathrm{~d}$ & $1,1.10^{3} \mathrm{c}$ & 7,0 & 7,0 & - & $2,6.10^{2} \mathrm{a}$ & $2.10^{1} \mathrm{a}$ \\
\hline $\begin{array}{l}\text { Critères } \\
\text { microbiologiques } \\
\text { (AFNOR 2000) }\end{array}$ & $10^{3}$ & 10 & 10 & 10 & 10 & 10 & 10 \\
\hline
\end{tabular}

Les chiffres portant la même lettre dans la même colonne ne sont pas significativement différents $(p<0,05), \quad$ - : Absence.

Tableau 4 : Flore potentiellement pathogène (ufc/g) dans les échantillons de poissons prélevés à différentes étapes du processus de fumage (Site2)

\begin{tabular}{|l|c|c|}
\hline Etapes de collectes & Anaérobies Sulfito Réducteurs & Salmonella spp. \\
\hline Réception & $6,0 \mathrm{a}$ & - \\
\hline Prétraitements & $4,0 \mathrm{a}$ & - \\
\hline Fumage & - & - \\
\hline Mise en vente & $1,0 \mathrm{a}$ & - \\
\hline Critères (AFNOR 2000) & $\mathbf{0 2}$ & Absence $\mathbf{2 5 g}$ \\
\hline
\end{tabular}

Les chiffres portant la même lettre dans la même colonne ne sont pas significativement différents $(p<0,05)$,

- : Absence

\section{CONCLUSION}

La présente étude réalisée au sud du Bénin révèle qu'll existe un risque potentiel de recontamination des poissons fumés, malgré l'application des bonnes pratiques de fabrication par les transformatrices et les effets conservateurs du fumage. Cette contamination provient essentiellement des conditions d'exposition et de vente des poissons fumés. De ce fait, une attention

particulière visant essentiellement la réduction de la charge microbienne des poissons doit être accordée à cette filière. L'application rigoureuse des règles d'hygiène tout au long du processus de fumage et de la mise en vente réduirait de façon significative la flore microbienne de contamination des poissons en augmentant leur durée de conservation.

\section{REMERCIEMENT}

Les auteurs remercient sincèrement le Département des Technologies Alimentaires de l'Ecole Polytechnique de I'Université d'Abomey-calavi (Bénin) pour son support financier dans la réalisation de ce travail.

\section{BIBLIOGRAPHIQUES}

AFSSA, 2009. Évaluation des risques liés à la présence de mycotoxines dans les chaînes alimentaires humaine et animale. Rapport final de Mars 2009, p. 308.

Anihouvi VB, Hounhouigan JD, Ayernor GS, 2005. La production et la commercialisation du Lanhouin, un condiment à base de poisson fermenté du
Golfe du Bénin. Cahiers Agricultures, 14(3) : 23330.

Anoh KP, 1998. Contribution à l'étude du réseau de distribution des ressources halieutiques marines en Côte d'Ivoire. Thèse 3eme cycle : géographie : Université de Côte d'Ivoire, faculté des arts et sciences humaines, Département de géographie, p. 323. 
CTA., 1990. Conserver et transformer le poisson- Guide Technique et méthodologie. Collection le point sur BP: 380, 6700 Wageningen, Pays Bas, p. 295.

Degnon RG, Dahouenon-ahoussi E, Adjou ES, Sohounhloue DCK, 2013. Transformation artisanale des crevettes (Penaeus spp) au sud du Bénin: évaluation des performances techniques des équipements et procédés de fumage. Nature \& Technologie, $08: 23-31$.

Diouf $F, 1992$. Contribution à l'étude de la qualité hygiénique des Aliments Vendus sur la Voie Publique (AVP) dans la région de Dakar Thèse : Méd. Vét. Dakar, p. 36.

Djinou HPAB, 2001. Etude de la qualité microbiologique du poisson fumé artisanalement en Côte d'Ivoire et destiné à l'exportation. Thèse : Méd. Vét., Dakar, p. 231.

FAO, 2000. United Nations Food and Agriculture Organization. FAO yearbook. Fishery statistics capture production. Vol. 86/1, 1998, pp. 99- 100.

FAO., 1979. Fumage du poisson. Rapport sur les pêches.-Rome : FAO., p. 47.

Food and Drug Administration, 1995. In Bacteriological Analytical Manuel 8th ed., Vibrio cholera, V. parahemoliticus, V. vulniticusand other Vibrio spp. AOAC International, Arlington, USA.

Gallot S, Fremy JM, 2006. Evaluation des risques lies à la présence des mycotoxines dans les chaines alimentaires humaines et animales. Rapport synthétique AFSSA, Paris, p. 25.

Gbaguidi A, Fiogbe E, 1999. Rapport national sur la pêche au Bénin, p. 20.

Goueu B, 2006. Contribution à l'étude de l'évolution de la qualité microbiologique du poisson fumé en Côte d'Ivoire et destiné à l'exportation. Thèse de doctorat en Médecine Vétérinaire de l'Ecole Inter Etats des Sciences de l'Université Cheikh Anta Diop de Dakar, pp. 55 - 127.

Huss HH, Reilly A, Embarek, BPK, 2000. Prevention and control of hazards in seafood. Food Control, 11 (2) : 149-156.

Joffin C, Joffin J-N, 2003. Microbiologie alimentaire. Biologie et Technique, 5è édition, CRDP Aquitaine, 212p.

Leclerc H, Schwarrtzbrod L, Dei-Cas E, 2002. Microbial agents associated with waterborne diseases. Crit. Rev. Microbiol., 28: 371 - 409.

OMS, 2003. Salubrité des Aliments et Santé : Analyse de la situation et perspectives, p. 19.
Oulaï SF, Koffi RA, Koussemon M, Djè M, Kakou C, Kamenan A, 2007. Evaluation de la qualité microbiologique des poissons Etmalosa fimbriata et Sardinella aurita fumés traditionnellement. Microbiologie et Hygiène Alimentaire, 19(55) :37-42.

Sutra L, Federighi M, Jouve J-L, 1998. Manuel de Bactériologie Alimentaire. 4 th ed, Polytechnica, Paris, France.

Tossou S, 2010. Rapport national d'évaluation de l'application des mesures sanitaires et phytosanitaires dans la commercialisation des produits de la pêche au Bénin, p. 8.

Untermann F., 1998. Microbial hazards of food. Food Control, $9: 119-126$

WHO/FAO, 1998. Forty-ninth meeting of the Joint Expert Committee on Food Additives. Food and Agricultural Organization of the United Nation. Rome, $140 \mathrm{p}$. 